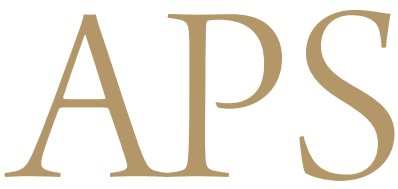

Archives of Plastic Surgery

\title{
Dual vascular free transverse rectus abdominis myocutaneous flap for hemifacial reconstruction in a vessel-depleted neck
}

\author{
Su-Hyun Lee, Hi-Jin You, Yun-Hwan Lee, Deok-Woo Kim \\ Department of Plastic Surgery, Korea University College of Medicine, Seoul, Korea
}

Cutaneous squamous cell carcinoma (SCC) is the second most common skin malignancy. This report describes the case of an unusual extensive SCC involving the whole hemiface, which required reconstruction with a combination of a dual vascular free transverse rectus abdominis muscle (TRAM) flap and a skin graft. A 79-year-old woman visited our hospital with multiple large ulcerated erythematous patches on her right hemiface, including the parieto-temporal scalp, bulbar and palpebral conjunctiva, cheek, and lip. A preliminary multifocal biopsy was performed in order to determine the resection margin, and the lesion was resected en bloc. Orbital exenteration was also performed. A free TRAM flap was harvested with preserved bilateral pedicles and was anastomosed with a single superior thyroidal vessel. The entire TRAM flap survived. The final pathological examination of the resected specimen confirmed that there was no regional nodal metastasis, perineural invasion, or lymphovascular involvement. The patient was observed for 6 months, and there was no evidence of local recurrence. Usage of a TRAM flap is appropriate for hemifacial reconstruction because the skin of the abdomen matches the color and pliability of the face. Furthermore, we found that the independent attachment of two extra-flap anastomoses to a single recipient vessel can safely result in survival of the flap.

Keywords Skin neoplasms / Free flaps / Squamous cell carcinoma of head and neck
Correspondence: Hi-Jin You Department of Plastic Surgery, Korea University Ansan Hospital, Korea University College of Medicine, 123 Jeokgeum-ro, Danwon-gu, Ansan 15355, Korea

Tel: +82-31-412-6810

Fax: +82-31-475-5074

E-mail: hijinyou@gmail.com

Received: July 14, $2019 \bullet$ Revised: October 31, $2019 \bullet$ Accepted: October 31, 2019

pISSN: 2234-6163 • elSSN: 2234-6171 • https://doi.org/10.5999/aps.2019.00927• Arch Plast Surg 2020;47:88-91

\section{INTRODUCTION}

Cutaneous squamous cell carcinoma (SCC) is the second most common malignant skin tumor [1]. It is associated with exposure to sunlight, exposure to ultraviolet radiation, burn scars, and chronic ulcers. The clinical presentation of this condition ranges from scaly plaques to ill-defined flat lesions with or without ulceration [2]. SCC commonly occurs on the face and therefore tends to be detected at an early stage. It is easily diag- nosed through physical examination and tissue biopsy [3]. Due to its infiltrative and metastatic features, early surgical treatment after diagnosis is crucial. If neglected, the lesions can become extensive.

Our unusual case of extensive and recurrent SCC covering the patient's whole hemiface involved reconstruction with the combination of a dual vascular free transverse rectus abdominis muscle (TRAM) flap and a skin graft. This report was approved by the Institutional Review Board of Korea University Ansan 
Hospital (IRB No. 2019AS0204). Written informed consent was obtained from the patient.

\section{CASE}

A 79-year-old woman visited our hospital with multiple large ulcerated erythematous patches on her right hemiface, including the parieto-temporal scalp, bulbar and palpebral conjunctiva, cheek, and lip (Fig. 1). The patient's history revealed that a small mass-like lesion had first appeared on her right malar area a few years ago, and she underwent punch biopsy 1 year prior to presenting to our hospital. The lesion was diagnosed as SCC, excised widely, and closed with a local flap at another hospital. The patient subsequently underwent 35 adjunctive radiotherapy sessions. However, 3 months after the completion of radiotherapy,

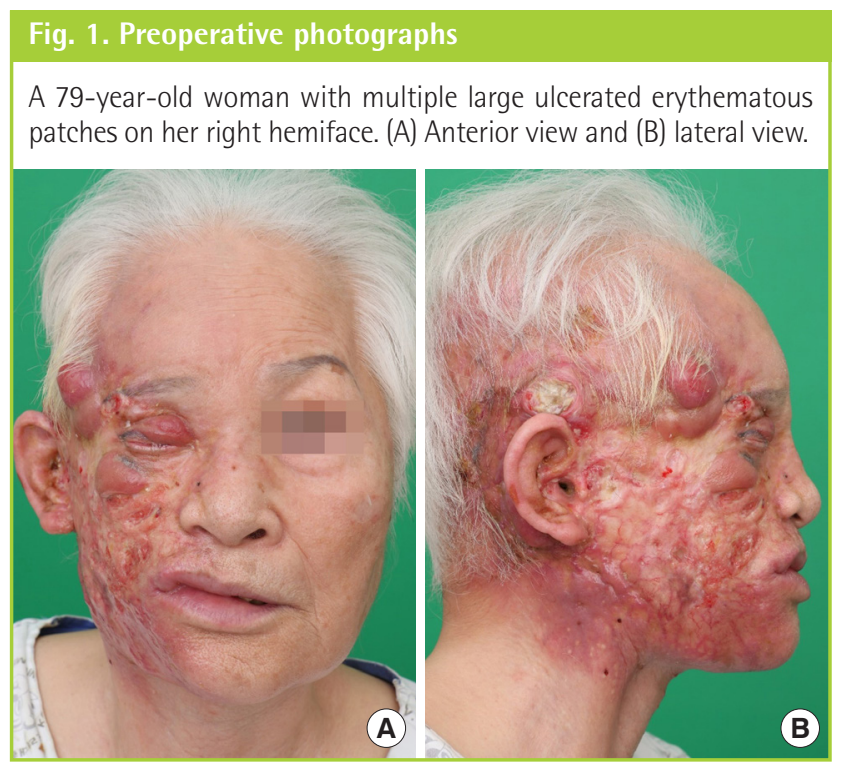

\section{Fig. 2. Intraoperative photographs}

Defect after wide excision. (A) Anterior view and (B) lateral view.
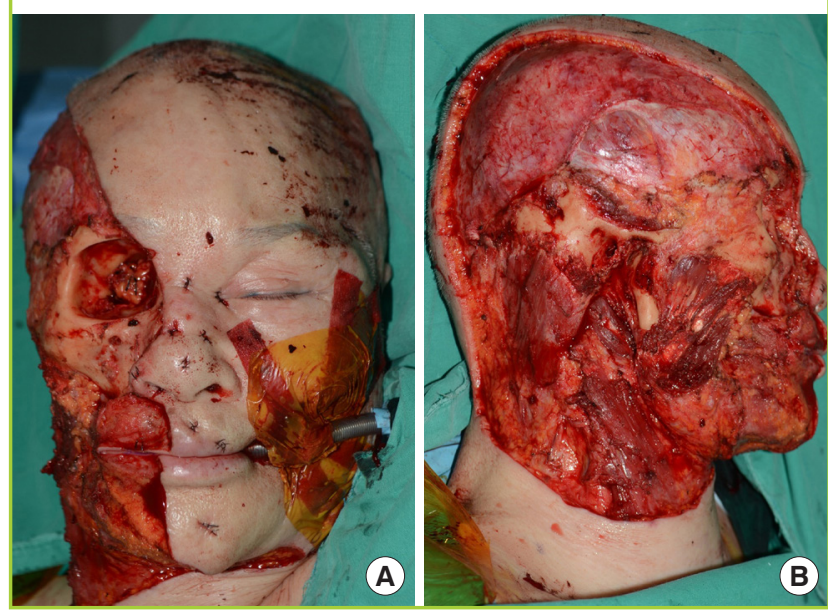

a larger ulcerative skin lesion combined with redness and swelling arose abruptly involving the entire right cheek, the same region as the therapeutic radiation field. The lesion was aggressive and rapidly spread throughout the skin, merging with the area of radiation dermatitis. Conservative management of the lesion, thought to be a radiation ulcer, was performed for the following 6 months. The patient visited a dermatologist at our institution for further management; punch biopsy revealed recurrent SCC. She was referred to the plastic surgery department for surgical management. No evidence of distant metastasis or bony abnormality was observed on magnetic resonance imaging, computed tomography, or positron emission tomography-computed tomography scans. Therefore, we decided to excise the entire lesion with a 6-mm resection margin as recommended.

The initial excision boundary was determined and drawn according to the preliminary biopsy results. The specimen was resected en bloc; frozen biopsy revealed tumor involvement of the deep margins of the paranasal area and eyeball. Further excision

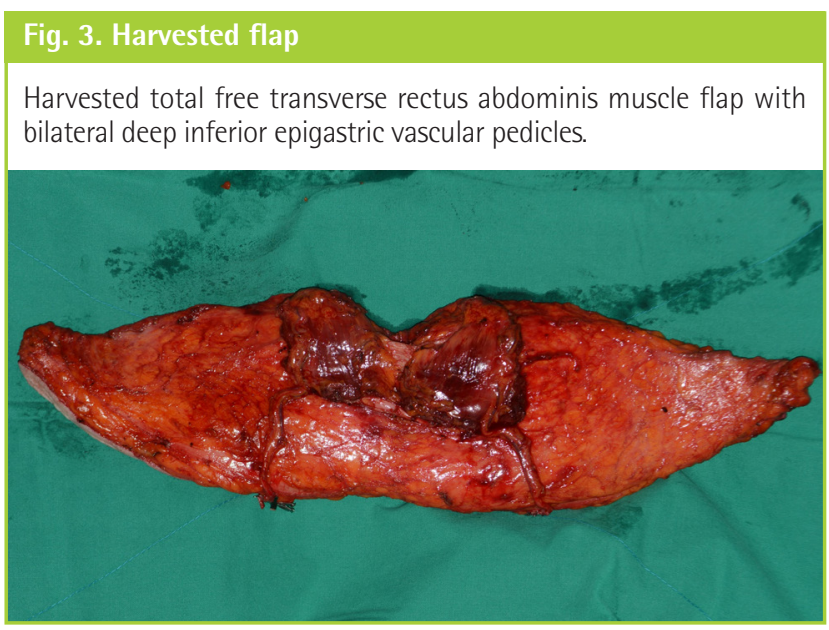

\section{Fig. 4. Intraoperative photograph}

Four end-to-side anastomoses for dual vascular supply (white arrowheads, superior thyroidal artery; white arrows, external jugular vein).

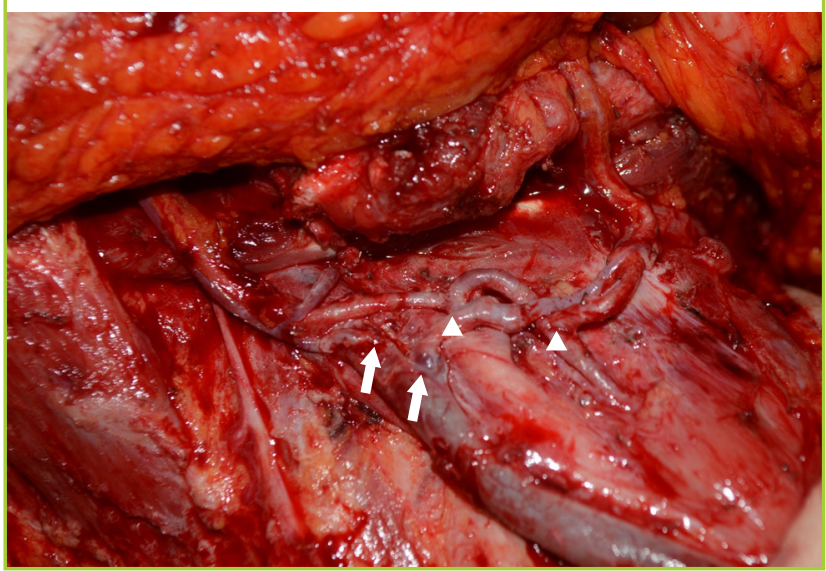




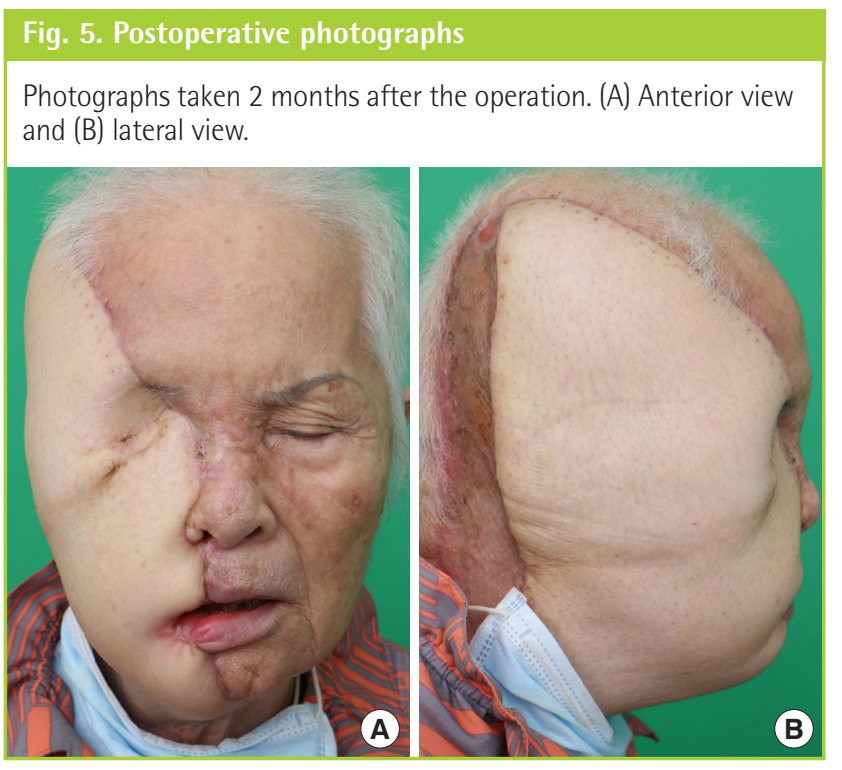

of the residual tumor in the paranasal bone and orbital exenteration were performed (Fig. 2). A total free TRAM flap with bilateral deep inferior epigastric vascular pedicles was harvested to cover the entire hemifacial defect (Fig. 3). Each pedicle was anastomosed end-to-side to the ipsilateral superior thyroidal artery and the external jugular vein (Fig. 4). The posterior scalp was covered with a split-thickness skin graft from the thigh. Pathological examination confirmed a moderately differentiated SCC with no regional nodal metastasis, perineural invasion, or lymphovascular involvement (T3NOM0). The operative site healed without complications, such as flap necrosis, and the grafted skin took successfully (Fig. 5). There was no evidence of local recurrence during the 6-month follow-up period.

\section{DISCUSSION}

Risk factors for SCC include exposure to radiation, immunosuppressed status, scars, and chronic wounds. Ultraviolet B light (290-320 nm), a component of sunlight, is highly carcinogenic and can cause DNA point mutations [2]. In addition to ultraviolet radiation, therapeutic radiation is another risk factor for SCC [2]. SCC is stratified into high- and low-risk SCC according to several factors [4]. The American Joint Committee on Cancer defined high-risk features for recurrence as a location of the lip, nose, or ear; a depth $>2 \mathrm{~mm}$; the presence of perineural invasion; and a poorly differentiated histology. The National Comprehensive Cancer Network recommends a 4-mm resection margin for low-risk SCC and a 6-mm resection margin for high-risk SCC $[4,5]$.

The lesion in our case started as a small nodular mass on the patient's right malar area, which was diagnosed as SCC, excised widely, and closed primarily, followed by 35 adjunctive radiotherapy sessions. After the conclusion of radiotherapy, a large ulcerative skin lesion combined with redness and swelling arose abruptly, involving the patient's entire right cheek. Initially, it was mistaken for a radiation ulcer due to its appearance and its involvement of an area coinciding with the therapeutic radiation field. However, the affected area did not disappear, but grew, even though radiotherapy had concluded. Since the lesion was confused with a radiation ulcer due to its appearance and location, diagnosis and treatment were delayed until it expanded to the point of covering the patient's hemiface. Physicians must keep in mind the possibility of recurrence and regularly follow up with patients who were previously treated for SCC $[4,5]$.

The entire lesion was excised with a safety margin, leaving a $16-21 \mathrm{~cm}$ defect on the right side of the patient's face, including the ear and temporoparietal scalp. We decided to use a bilateral TRAM flap for reconstruction. Two separate extra-flap end-toside anastomoses were performed, as separate anastomoses can reduce the risk of total flap loss relative to when an intra-flap anastomosis is used [6].

Many reports have described using bipedicled TRAM flaps or deep inferior epigastric perforator flaps for postmastectomy breast reconstruction. However, reconstructions of head and neck defects using those techniques are very rare. Chung et al. [7] reported a rare case of basaloid SCC on the hemiface. The final hemifacial defect was similar to the defect in our case, but the TRAM flap used in that case was divided in half. In our case, we used a bilateral, bipedicled TRAM flap without division. To our knowledge, ours is the first case in which a bilateral, bipedicled TRAM flap was applied and two extra-flap anastomoses to one recipient vessel were performed for the reconstruction of a craniofacial defect.

Many options are available to cover large defects, including TRAM flaps, latissimus dorsi myocutaneous flaps, and anterolateral thigh flaps. Except in the cases of extremely obese patients, we believe it appropriate to rebuild the hemiface with a TRAM flap. The thickness of the subcutaneous fat is adequate and, more importantly, the skin quality of the TRAM flap closely matches the color and pliability of the facial skin. Furthermore, the TRAM flap donor site exhibits low morbidity and can heal rapidly because it can be closed primarily [8]. In contrast, the amount of skin available for a latissimus dorsi myocutaneous flap or an anterolateral thigh flap is limited, and the skin quality of those regions is very different from that of the face. Although a case report has described the reconstruction of a large head and neck defect with a tripedicled, near-total thigh flap using the supercharging technique [9], a skin graft was required to cover the donor site in that case. Skin grafting takes a relatively 
long time to heal and can induce scar contracture and gait impairment. In latissimus dorsi myocutaneous flaps, the skin quality is very different from that of the face, and complications such as shoulder impairment, chronic back pain, or seroma can arise.

Reconstruction of an extensive facial defect is challenging. Usage of a dual vascular pedicled TRAM flap takes advantage of the largest available skin area and abundant subcutaneous fat. Therefore, the use of such a flap is suitable for reconstructing extensive defects of the craniofacial area with minimal donor site morbidity. Moreover, the skin thickness, color, and texture of the flap closely match the skin of the face. These characteristics contribute to satisfactory clinical outcomes, and the use of this technique can save operating time and cost in the coverage of very large defects. In this case, we performed two extra-flap anastomoses to the same recipient vessel, a technique that resulted in flap survival. This technique is similar to the turbocharging technique in that the entire TRAM flap is supplied by the same source vessel. The turbocharging technique regards the contralateral pedicle as a minor pedicle used to improve transumbilical perfusion. However, our technique is slightly different in that both pedicles are considered major pedicles and are anastomosed independently. Therefore, we believe that the circulation provided by a total TRAM flap is superior to that provided by the turbocharging technique.

This case is meaningful in that it involves a successful reconstruction accomplished by connecting two pedicles to the same recipient vessel using a bipedicled, bilateral TRAM flap on a large craniofacial defect encompassing half of the face in the temporoparietal scalp area. This report provides guidance for cases in which previous radiotherapy left only one recipient vessel available or in which few recipient vessels are available due to multiple prior flap operations.

\section{NOTES}

\section{Conflict of interest}

No potential conflict of interest relevant to this article was reported.

\section{Ethical approval}

The study was approved by the Institutional Review Board of Korea University Ansan Hospital (IRB No. 2019AS0204) and performed in accordance with the principles of the Declaration of Helsinki. Written informed consent was obtained.

\section{Patient consent}

The patient provided written informed consent for the publication and the use of her images.

\section{Author contribution}

Conceptualization: You HJ. Data curation: Lee YH. Formal analysis: Kim DW. Methodology: You HJ. Project administration: You HJ. Visualization: You HJ. Writing - original draft: Lee SH. Writing - review \& editing: Lee SH. Approval of final manuscript: all authors.

\section{ORCID}

Su-Hyun Lee https://orcid.org/0000-0001-5846-392X

Hi-Jin You https://orcid.org/0000-0002-9997-2736

Yun-Hwan Lee https://orcid.org/0000-0002-0881-7926

Deok-Woo Kim https://orcid.org/0000-0003-3382-560X

\section{REFERENCES}

1. Oh CM, Cho H, Won YJ, et al. Nationwide trends in the incidence of melanoma and non-melanoma skin cancers from 1999 to 2014 in South Korea. Cancer Res Treat 2018;50: 729-37.

2. Rudolph R, Zelac DE. Squamous cell carcinoma of the skin. Plast Reconstr Surg 2004;114:82e-94e.

3. Thomas DJ, King AR, Peat BG. Excision margins for nonmelanotic skin cancer. Plast Reconstr Surg 2003;112:57-63.

4. Mendez BM, Thornton JF. Current basal and squamous cell skin cancer management. Plast Reconstr Surg 2018;142: 373e-387e.

5. Connolly KL, Nehal KS, Disa JJ. Evidence-based medicine: cutaneous facial malignancies: nonmelanoma skin cancer. Plast Reconstr Surg 2017;139:181e-190e.

6. Bota O, Spindler N, Sauber J, et al. Double-pedicled free deep inferior epigastric perforator flap for the coverage of thigh soft-tissue defect. Plast Reconstr Surg Glob Open 2017;5: e1372.

7. Chung JH, You HJ, Hwang NH, et al. Soft tissue reconstruction for basaloid squamous cell carcinoma on the hemiface. Arch Plast Surg 2016;43:615-8.

8. Slater JC, Sosin M, Rodriguez ED, et al. Bilateral, Bipedicled DIEP flap for staged reconstruction of cranial deformity. Craniomaxillofac Trauma Reconstr 2014;7:313-7.

9. Sokoya M, Deleyiannis FW. A triple pedicle, near-total thigh flap superchargedwith the flow-through technique. Eplasty 2016;16:e4. 